\title{
Phase Equilibria in High Energy Density PVDF-Based Polymers
}

\author{
V. Ranjan and L. Yu \\ Center for High Performance Simulation and Department of Physics, North Carolina State University, \\ Raleigh, North Carolina 27695-7518, USA \\ Marco Buongiorno Nardelli and J. Bernholc \\ Center for High Performance Simulation and Department of Physics, North Carolina State University, \\ Raleigh, North Carolina 27695-7518 \\ and Computer Science and Mathematics Division, Oak Ridge National Laboratory, Oak Ridge, Tennessee 37830, USA
}

(Received 15 March 2007; published 25 July 2007)

\begin{abstract}
The phase diagrams of polyvinylidene fluoride (PVDF) and its copolymers with chlorotrifluoroethylene (CTFE) are investigated by first-principles calculations. Both PVDF and dilute P(VDF-CTFE) prefer nonpolar structures at zero field, but transform to a polar phase below the breakdown field. The critical field decreases with increasing CTFE content, facilitating the transformation. In disordered P(VDFCTFE), a distribution of concentrations leads to a range of polar transitions, resulting in ultrahigh energy density. These results explain well experimental observations of very high-energy density in P(VDFCTFE).
\end{abstract}

DOI: 10.1103/PhysRevLett.99.047801

PACS numbers: 61.41.+e, 71.15.Nc, 77.22.-d

Polyvinylidene fluoride (PVDF) and its copolymers are well-known ferroelectric polymers that exhibit excellent electromechanical properties [1-4] and are also of substantial recent interest as candidates for high-performance, high-energy-density capacitors. They exist in two main phases, the nonpolar $\alpha$ phase and the polar $\beta$ phase. The existence of two phases provides an avenue for manipulating the electrical and electromechanical properties of these polymers, potentially enabling novel applications. In pure PVDF, an ordered $\beta$ phase is obtained by either mechanical rolling or stretching [5] or application of a very strong electric field [6]. Once $\beta$ PVDF is formed, the dipoleordering forces are so strong that this phase melts before it can undergo a ferroelectric-to-paraelectric phase transition. However, in copolymers of VDF and trifluoroethylene TeFE $\left[-\mathrm{CF}_{2}-\mathrm{CFH}\right]$ with about $50 \%-80 \% \mathrm{VDF}$, the $\beta$ phase is formed directly and a ferroelectric phase transition has been observed below the melting point [5].

These phase transitions are associated with an increased dielectric constant and energy density. The energy density of a dielectric material, defined as the maximum energy stored in the material when an electric field is applied, is defined as $\xi=\int_{0}^{D\left(E_{\max }\right)} E d D$, where $E$ is the electric field, $D$ is the electric displacement, and the integration proceeds up to the maximum applied field $E_{\max }$ just below the breakdown field. Recent experimental work [7] identified a copolymer of VDF-chlorotrifluoroethylene, P(VDFCTFE), as having an unusually high-energy density, in excess of $17 \mathrm{~J} / \mathrm{cm}^{3}$, much higher than that of polypropelene, which has the highest energy density among the previously investigated polymers $\left(4 \mathrm{~J} / \mathrm{cm}^{3}\right)$. In P(VDFCTFE), the energy density increases dramatically with the electric field and does not saturate until the breakdown field is reached. This behavior is very different from that of pure PVDF, or the commonly used P(VDF-TeFE) copolymers, where energy density increases linearly with electric field due to early saturation of polarization [7]. The difference in behaviors of the two polymers was attributed to a nonpolar to polar phase transition in P(VDF-CTFE), since its films were grown in the nonpolar phase.

In this Letter, the phase diagram of the P(VDF-CTFE) polymer is investigated by ab initio methods as a function of the applied electric field and CTFE concentration. It is shown that ordered phases of P(VDF-CTFE) with welldefined concentrations exhibit sharp phase transformations and polarization that saturates at very low fields for both $\alpha$ and $\beta$ phases. The saturation of polarization would lead to saturation in the displacement field and a low energy density. This is in sharp contrast to the experimental data. However, if a multidomain disordered structure is assumed, we show that it leads to a strongly increasing displacement field and a large dielectric constant that saturates only at very high fields, in agreement with the experimental results. We also show that by controlling the distribution of nanostructures with different critical fields for the para- to ferroelectric transition one can substantially enhance the energy density and/or design nanostructured materials with desired dielectric properties. Furthermore, the proposed procedure is not limited to polymers and can be used for any mixture of materials that exbibit para- to ferroelectric transition and where the critical electric field for the transition varies with the concentration of one of the constituents. For P(VDF-CTFE), slightly reducing the amount of disorder would lead to significantly greater energy density.

The calculations are performed using density-functional theory (DFT) with ultrasoft pseudopotentials and PerdewBurke-Ernzerhof exchange-correlation functional [8] as 
implemented in the PWSCF code [9] with a cutoff energy of $35 \mathrm{Ry}$ and a $2 \times 4 \times 4$ Monkhorst-Pack $k$-point grid [8]. The Berry phase approach [10] is used to compute polarization $\mathbf{P}$ and the Born effective charges $Z_{i}^{\star}$ for each of the ions $i$ are obtained using density functional perturbation theory [11]. Following $\mathrm{Fu}$ and Bellaiche [12] the term $-e Z_{i}^{\star} E$ is added to the Hellman-Feynman forces $\mathbf{f}_{i}$, where $e$ is the elementary charge. The atomic structure is relaxed until the total force on each atom is close to zero, i.e., until $\mathbf{f}_{i}=-e Z_{i}^{\star} E[12-14]$.

The structure of PVDF is obtained by minimizing the electric enthalpy (free energy under electric field) $F$ per unit cell,

$$
F(E)=U-\Omega \mathbf{P E},
$$

where $U$ is the internal energy obtained from a Kohn-Sham functional, and $\mathbf{P}=\mathbf{P}_{\text {ion }}+\mathbf{P}_{\mathrm{el}}$ is the total (ionic plus electronic) macroscopic polarization. Within this scheme, the field dependence of $\mathbf{f}_{i}$ and $Z_{i}^{\star}$ is neglected, because they are computed at $E=0$. In particular, the results are rigorously correct only to first order with regard to fieldinduced lattice displacements [14] and electronic nonlinearities are not considered. However, using the linear response perturbation theory, we found that the Born effective charges change by less than $8 \%$ at $1000 \mathrm{MV} / \mathrm{m}$, compared to their zero-field values. Therefore, the FuBellaiche scheme is sufficiently accurate for studying field-induced phase transitions in PVDF and related polymers.

Figure 1 depicts the bulk $\alpha$ (nonpolar) and $\beta$ (polar) PVDF structures [1]. Both the $\alpha$ and $\beta$ phases have the

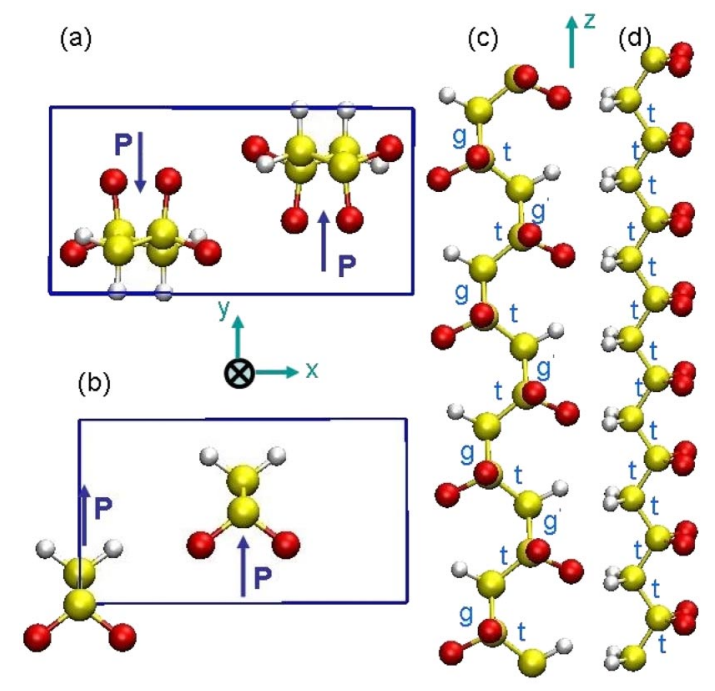

FIG. 1 (color online). The unit cell of bulk PVDF in the (a) $\alpha$ and (b) $\beta$ phases. The carbon, fluorine, and hydrogen atoms are yellow, red, and white, respectively. The $\alpha$ phase is antipolar, since dipoles in the alternate chains cancel, while in the $\beta$ phase they are parallel to each other. The panels (c) and (d) show the trans-gauche arrangement in the $\alpha$ phase and all trans arrangement in the $\beta$ phase, respectively. same repeat monomeric unit, $-\left(\mathrm{CF}_{2}-\mathrm{CH}_{2}\right)-$. The chain conformation in the $\alpha$ phase is trans-gauche-transgauche', whereas it is all trans in the $\beta$ phase. The $\alpha$ phase unit cell consists of two polymer chains arranged in an antipolar fashion, resulting in zero net polarization. In the $\beta$ phase, the charge dipoles of the two chains are parallel and thus the structure is polar. An earlier $a b$ initio calculation [4] obtained a substantial polarization of $0.18 \mathrm{C} / \mathrm{m}^{2}$ in the $\beta$ phase.

We found that the total energy of $\alpha$-PVDF is $23 \mathrm{meV}$ per carbon atom lower than the one of $\beta$-PVDF making $\alpha$-PVDF the low-temperature stable phase in agreement with other works $[3,5]$. The Born effective charges for each atom are obtained from the zero-field ground state structure of the $\alpha$ and $\beta$ PVDF. Both phases are then subjected to an electric field and their free energy is minimized following the scheme described above.

Figure 2(a) depicts the change in free energy, $F(E)$, for both phases as the electric field is varied from 0 to $1000 \mathrm{MV} / \mathrm{m}$. Because of negligible polarization in the $\alpha$ phase, its free energy remains approximately constant in the entire field range. In contrast, the free energy of the polar $\beta$ phase decreases with the field and $\beta$ becomes the stable phase at fields above $\sim 800 \mathrm{MV} / \mathrm{m}$. The calculated critical field is similar to the ones reported in the literature, where it is usually quoted as $\approx 500 \mathrm{MV} / \mathrm{m}[6]$. Figure $2(\mathrm{~b})$ shows the electric displacement $D=E+4 \pi P$ as a function of the applied electric field for both $\alpha$ and $\beta$ phases. The displacement field changes relatively little with the field in each phase, but the change in the stable phase at the critical field of $\sim 800 \mathrm{MV} / \mathrm{m}$ results in a large discontinuous change in $D$. The vertical line marks the transformation from the $\alpha$ to the $\beta$ phase. The volume change at the transition is discontinuous, $4.5 \%$, which is a signature of

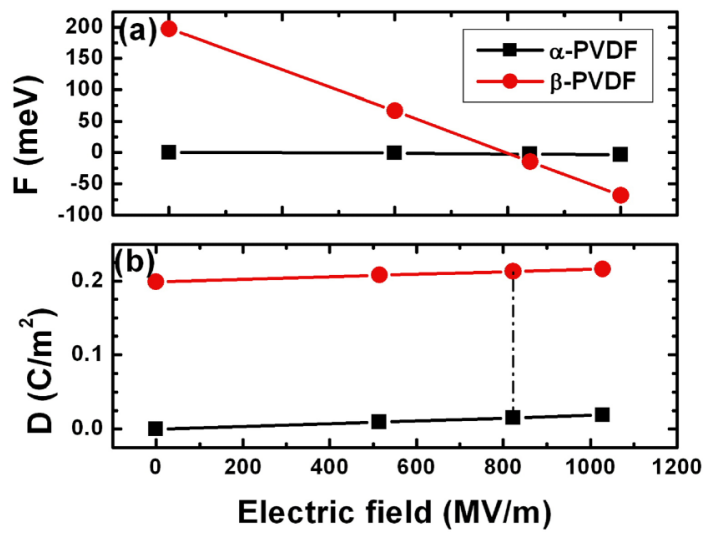

FIG. 2 (color online). (a) Free energy as a function of the electric field for $\alpha$ - and $\beta$-PVDF. $\alpha$-PVDF is lower in energy at zero field, but $\beta$-PVDF becomes the favored phase at $\sim 800 \mathrm{MV} / \mathrm{m}$. (b) Electric displacement field $D$ versus applied electric field for $\alpha$ - and $\beta$-PVDF. The vertical line at $\sim 800 \mathrm{MV} / \mathrm{m}$ marks the transition from $\alpha$ - to $\beta$-PVDF, when $D$ undergoes a discontinuous change. 
first-order phase transition. Therefore, nucleation of the $\beta$ phase is required at the transition.

We also investigate the $\alpha$ and $\beta$ phases of P(VDFCTFE) with varying concentrations of CTFE. These calculations are carried for ordered structures for concentrations ranging from $10 \%$ to $50 \%$, using a single CTFE monomer in supercells of the appropriate sizes. Figure 3(a) depicts the difference in total energy between the $\alpha$ and $\beta$ phases as a function of CTFE concentration. For CTFE concentrations below $17 \%$, the $\alpha$ phase is more stable than the $\beta$ phase. Since this difference decreases with the concentration of CTFE, the transformation to the $\beta$ phase becomes progressively easier as the critical concentration is approached. Above $17 \%$, the $\beta$ phase is preferred and no field-induced transformation should occur.

The electric field needed to transform from the $\alpha$ to the $\beta$ phase is not only dependent on the total energy differences, but also on polarization. The total polarization for the $\beta$ phase decreases as the CTFE concentration increases, from $0.2 \mathrm{C} / \mathrm{m}^{2}$ for pure PVDF to $0.06 \mathrm{C} / \mathrm{m}^{2}$ for 50\% CTFE; see Fig. 3(b).

Chu et al. [7] observed that their P(VDF-CTFE) 91/9\% polymer is in the $\alpha$ phase, both as grown and after uniaxial stretching. (The stretching improves the breakdown field and thus the maximum achievable energy density.) This is in agreement with the results shown in Fig. 3(a). In the experiment, both the displacement field and the energy density increase smoothly with the field up to the maximum breakdown field of $\sim 550 \mathrm{MV} / \mathrm{m}$. The authors [7] suggest that the high-energy density is due to a reversible change in conformation between the nonpolar and polar phases of P(VDF-CTFE), which could explain the large increase in $D$ and the lack of its saturation. However, the above theoretical results for ordered dilute P(VDF-CTFE) show that $D$ in the $\alpha$ phase is very small and cannot reach the observed values of up to $\sim 0.08 \mathrm{C} / \mathrm{m}^{2}$; see Fig. 4(a). On the other hand, $D$ in the $\beta$ phase is substantially larger than

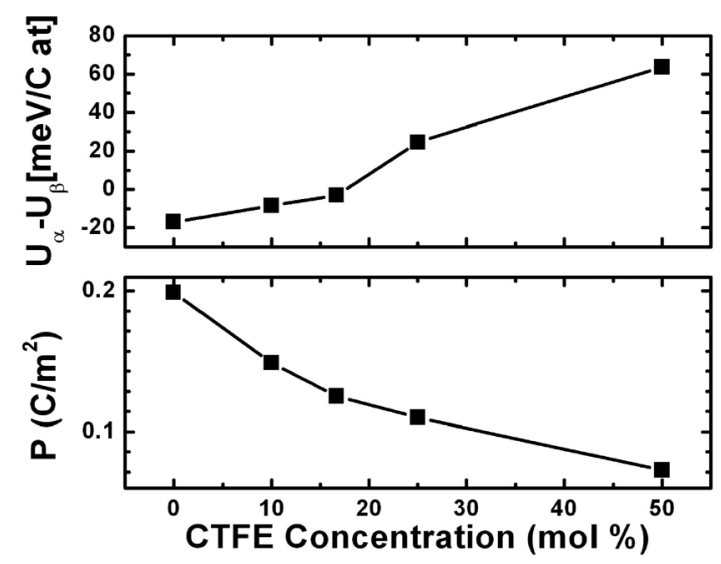

FIG. 3. Calculated thermodynamic properties P(VDF-CTFE) as functions of CTFE concentration: (a) difference between the total energies of $\alpha$ and $\beta$ phases, (b) polarization in the $\beta$ phase ( $\alpha$ phase has zero polarization). observed, especially for the lower fields. Furthermore, a thermodynamic transition to the $\beta$ phase should result in an abrupt change in polarization, which serves as the order parameter, and therefore in $D=E+4 \pi P$. This abrupt change is not observed in experiment.

Clearly, the above results for ordered P(VDF-CTFE) cannot explain the experimental data. However, while $\mathrm{x}$ ray diffraction data [7] show the major peaks associated with the $\alpha$ phase, the background scattering is large, indicating substantial disorder and lack of full crystallinity. To account for these effects, we consider a model in which the sample is assumed to consist of "nanodomains", each with a slightly different concentration of CTFE. Taking a Gaussian distribution of these domains [15], the probability $n(x)$ of a P(VDF-CTFE) domain with concentration $x \%$ becomes

$$
n(x)=\frac{1}{\sqrt{2 \pi} \sigma} \exp \left[-\frac{\left(x-x_{0}\right)^{2}}{2 \sigma^{2}}\right]
$$

where $x_{0}=0.1$, corresponding to the average CTFE density of $10 \%$ in the experiment. The width of the distribution, $\sigma$, is treated as an adjustable parameter, because the disorder in the experimental samples was not characterized. In the low concentration regime of the experiment, the $\alpha$ phase should dominate in each of the nanodomains. However, depending on the actual concentration, each nanodomain will transform to the polar $\beta$ phase at a different field value, which can be estimated from $U_{\alpha}-U_{\beta}=$ $-\Omega P_{\beta} E_{\text {crit }}$. Using the results of Fig. 3(a) and accounting for the different critical field in each nanodomain one obtains the total polarization at a given field as

$$
P(E)=\int_{0}^{1} d x \int_{0}^{E} d E^{\prime} P_{\beta}(x) n(x) \delta\left[E^{\prime}-E_{\text {crit }}(x)\right]
$$

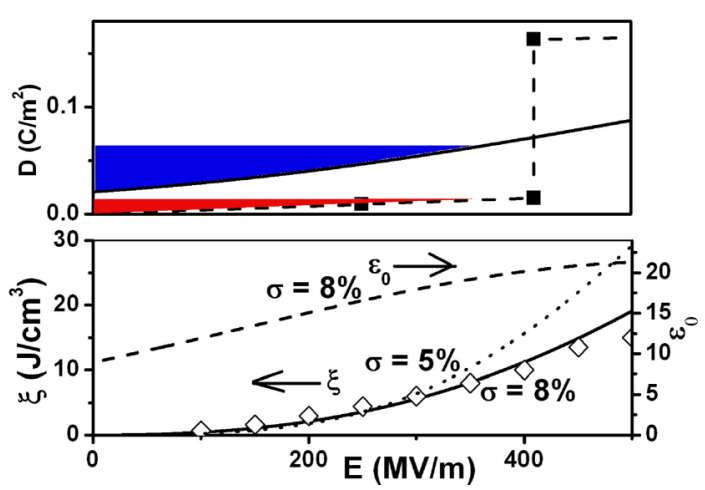

FIG. 4 (color online). (a) Displacement field as a function of the electric field for ordered PVDF-CTFE 90/10 mol\% (dashed line) and for a Gaussian distribution of nanodomains in P(VDFCTFE) centered at 90/10 mol\%, with $\sigma=8 \%$ (solid line). The blue-shaded area is the energy density for the disordered structure at $350 \mathrm{MV} / \mathrm{m}$, whereas the red-shaded area is for the ordered structure. (b) The dielectric constant (dashed line) and the energy density (solid line) for $\sigma=8 \%$. The diamonds are experimental results from Ref. [7]. The dotted line is the energy density for $\sigma=5 \%$. See text. 
where $n(x)$ is the normalized probability of having a domain with concentration $x$. The contribution to polarization of the domains that remain in the $\alpha$ phase turns out to be small enough that it can be neglected. The resulting displacement field increases with the electric field and agrees well with experiment for the broadening parameter $\sigma$ of $8 \%$; see Fig. 4(a). The increase in $D$ is due to conversion of a growing number of nanodomains to the $\beta$ phase until the peak of the distribution is reached (here at $10 \%$ ). Beyond this peak, the number of nanodomains that convert with an increase in the field decreases, resulting in decrease in the slope of $D$. The broadening parameter also partially mimics kinetic effects in each domain, since the need for nucleation of the $\beta$ phase should result in a distribution of effective critical fields.

The dielectric constant, given by $1+4 \pi d P(E) / d E$, is shown in Fig. 4(b). At first it increases with the field, as more domains are converted to the $\beta$ phase, but it starts to decrease rapidly when the displacement field starts to saturate. For a system that is energetically much closer to the $\beta$ phase, one would expect a high initial dielectric constant, which then decays as $\sim 1 / E$ after its polarization reaches saturation. This was observed for $\mathrm{P}(\mathrm{VDF}-\mathrm{TrFE})$ $75 / 25 \%$ [7], which has a dielectric constant of $\sim 50$ at the field of $50 \mathrm{MV} / \mathrm{m}$, but only $\sim 12$ at $400 \mathrm{MV} / \mathrm{m}$. In contrast, for the P(VDF-CTFE) model centered at $90 / 10 \%$, the calculated dielectric constant progressively increases with the field, until the displacement field reaches saturation. In this case, saturation occurs at fields $>500 \mathrm{MV} / \mathrm{m}$, which is close to the breakdown field. The energy density $\xi$, calculated as the area between the displacement field curve and the vertical axis in Fig. 4(a) (shown shaded at $E=$ $350 \mathrm{MV} / \mathrm{m}$ ), is substantial even at lower fields, which is necessary for applications. It reaches $\sim 20 \mathrm{~J} / \mathrm{cm}^{3}$ at $500 \mathrm{MV} / \mathrm{m}$, in reasonable agreement with the experimental value of $17 \mathrm{~J} / \mathrm{cm}^{3}$ [7]. In contrast, the energy density of the ordered structure is very small at low fields, but it would abruptly jump at the transition field. The accompanying abrupt change in volume is certainly detrimental for applications. If the disorder is reduced to $\sigma=5 \%$ [dotted line in Fig. 4(b)], the energy density just below the breakdown field reaches $29 \mathrm{~J} / \mathrm{cm}^{3}$. Another way to enhance substantially the energy density would be to reduce the CTFE concentration somewhat, say to $5 \%$, which would enhance both the saturation polarization and the saturation field, while still keeping the saturation field below the breakdown field. The above results clearly show that one can tune the energy density of P(VDF-CTFE) and related systems by substantial amounts through adjusting the concentration of CTFE and controlling the disorder. Furthermore, the relation between concentration, disorder, and the energy density uncovered here for the P(VDFCTFE) system is general and can be exploited in other material systems through, e.g., controlled deposition of nanodomains with different critical fields for the para- to ferroelectric transition.
In summary, we investigated the phase diagram of PVDF and P(VDF-CTFE) as a function of the applied electric field. Within the $a b$ initio density functional theory, crystalline PVDF and ordered P(VDF-CTFE) up to $17 \%$ CTFE prefer the nonpolar $\alpha$ phase. In sufficiently high electric fields, the polar $\beta$ phase becomes thermodynamically preferred, due to the $-P E_{\text {applied }}$ term. For P(VDFCTFE), the energy difference between the $\alpha$ and $\beta$ phases decreases with increase of the CTFE content, while the volume of the polymer increases. Both factors facilitate the transition to the $\beta$ phase with the applied electric field. For disordered samples, this transition is gradual, resulting in a gradual increase in the displacement field and a large energy density. These results are in good agreement with the experimental data and explain the origin of the observed ultrahigh capacitance in P(VDF-CTFE) films. Furthermore, these effects are general and suggest a systematic route, not limited to polymers, for obtaining nanostructured materials with high-energy density. Such materials could find widespread use in energy storage, power conditioning, and integrated circuitry.

This work was supported by the Office of Naval Research. The calculations were carried out at DOD-ARSC and the NCCS-ORNL. We thank Professor Q. Zhang for sharing and discussing his results prior to publication.

[1] A. J. Lovinger, Science 220, 1115 (1983).

[2] H. S. Nalwa, Ferroelectric Polymers: Chemistry, Physics, and Applications (Marcel Dekker, New York, 1995).

[3] H. Su, A. Strachan, and W. A. Goddard, III, Phys. Rev. B 70, 064101 (2004).

[4] S. M. Nakhmanson, M. Buongiorno Nardelli, and J. Bernholc, Phys. Rev. Lett. 92, 115504 (2004).

[5] G. A. Samara, Solid State Physics (Academic, San Diego, 2001), Vol. 56, p. 387.

[6] G. T. Davis, J.E. McKinney, M. G. Broadhurst, and S. C. Roth, J. Appl. Phys. 49, 4998 (1978).

[7] B. Chu, X. Zhou, K. Ren, B. Neese, M. Lin, Q. Wang, F. Bauer, and Q. M. Zhang, Science 313, 334 (2006).

[8] R. M. Martin, Electronic Structure (Cambridge University Press, Cambridge, England, 2004).

[9] S. Baroni, A. Dal Corso, S. de Gironcoli, and P. Giannozzi et al., http://www.pwscf.org.

[10] R. D. King-Smith and D. Vanderbilt, Phys. Rev. B 47, 1651 (1993).

[11] S. Baroni, S. de Gironcoli, A. Dal Corso, and P. Giannozzi, Rev. Mod. Phys. 73, 515 (2001).

[12] H. Fu and L. Bellaiche, Phys. Rev. Lett. 91, 057601 (2003).

[13] N. Sai, K. M. Rabe, and D. Vanderbilt, Phys. Rev. B 66, 104108 (2002).

[14] A. Antons, J. B. Neaton, K. M. Rabe, and D. Vanderbilt, Phys. Rev. B 71, 024102 (2005).

[15] V. Ranjan, V. A. Singh, and G. C. John, Phys. Rev. B 58, 1158 (1998). 